\title{
Selective Retinoic Acid Receptor $\gamma$ Agonists Promote Repair of Injured Skeletal Muscle in Mouse
}

\author{
Agnese Di Rocco, * Kenta Uchibe, ${ }^{*}$ Colleen Larmour, ${ }^{*}$ Rebecca Berger, ${ }^{*}$ Min Liu, ${ }^{\dagger}$ Elisabeth R. Barton, ${ }^{\ddagger}$ and Masahiro Iwamoto*
}

From the Translational Research Program in Pediatric Orthopaedics, ${ }^{*}$ The Children's Hospital of Philadelphia Research Institute, Philadelphia; and the Departments of Physiology ${ }^{\dagger}$ and Anatomy and Cell Biology, ${ }^{\ddagger}$ School of Dental Medicine, University of Pennsylvania, Philadelphia, Pennsylvania

\author{
Accepted for publication \\ May 19, 2015. \\ Address correspondence to \\ Masahiro Iwamoto, Ph.D., \\ D.D.S., The Children's Hospital \\ of Philadelphia, 3615 Civic \\ Center Blvd, Abramson \\ Research Center 910A, Phila- \\ delphia, PA 19104. E-mail: \\ iwamotom@email.chop.edu.
}

\begin{abstract}
Retinoic acid signaling regulates several biological events, including myogenesis. We previously found that retinoic acid receptor $\gamma(\operatorname{RAR} \gamma)$ agonist blocks heterotopic ossification, a pathological bone formation that mostly occurs in the skeletal muscle. Interestingly, RAR $\gamma$ agonist also weakened deterioration of muscle architecture adjacent to the heterotopic ossification lesion, suggesting that RAR $\gamma$ agonist may oppose skeletal muscle damage. To test this hypothesis, we generated a critical defect in the tibialis anterior muscle of 7-week-old mice with a cautery, treated them with RAR $\gamma$ agonist or vehicle corn oil, and examined the effects of RAR $\gamma$ agonist on muscle repair. The muscle defects were partially repaired with newly regenerating muscle cells, but also filled with adipose and fibrous scar tissue in both RAR $\gamma$-treated and control groups. The fibrous or adipose area was smaller in RAR $\gamma$ agonist-treated mice than in the control. In addition, muscle repair was remarkably delayed in RAR $\gamma$-null mice in both critical defect and cardiotoxin injury models. Furthermore, we found a rapid increase in retinoid signaling in lacerated muscle, as monitored by retinoid signaling reporter mice. Together, our results indicate that endogenous RAR $\gamma$ signaling is involved in muscle repair and that selective RAR $\gamma$ agonists may be beneficial to promote repair in various types of muscle injuries. (Am J Pathol 2015, 185: 2495-2504; http://dx.doi.org/10.1016/j.ajpath.2015.05.007)
\end{abstract}

Skeletal muscles can be injured because of a variety of reasons, including overuse, trauma, infections, and loss of blood circulation. In general, skeletal muscles have adequate repair capacity and quickly recover to full functionality after minor injury. However, repair of severely damaged muscle is challenging and may not resolve completely, even after an extended period. This is especially true in elderly persons or in individuals with repetitive torn or ruptured muscle injury. ${ }^{1}$ As a consequence of incomplete repair, damaged muscles accumulate connective scar tissue, leading to tissue stiffness and future damage, and ultimately decreasing muscle functionality. ${ }^{2,3}$ Current therapies, such as massage, ultrasound, ${ }^{4}$ hyperbaric oxygen delivery, and pain relievers, ${ }^{5}$ are mostly palliative. Therefore, an effective treatment method needs to be developed. Several biological drugs, such as insulin-like growth factor-1 and myostatin inhibitors, which are potent inducers of myogenesis, are expected to improve muscle regeneration in systemic myopathies, including muscular dystrophy. ${ }^{6,7}$ These biological agents have been studied in clinical trials (eg, NCT01207908 for insulin-like growth factor-1) and may also facilitate muscle repair. ${ }^{8-10}$

Heterotopic ossification (HO) is a pathological bone formation that mostly occurs in the skeletal muscle. More than $10 \%$ of patients undergoing invasive surgeries ${ }^{11-13}$ and a staggering $65 \%$ of seriously wounded soldiers ${ }^{14}$ develop HO. Current treatment options are nonsteroidal

Supported by The Muscular Dystrophy Association grant MDA255541 and National Institute of Arthritis and Musculoskeletal and Skin Diseases, NIH, grant AR056837.

Disclosures: M.I. received compensation from Clementia Pharmaceutical Company for consulting services. M.I. is one of the inventors named on a related patent application "Composition and Method for Muscle Repair and Regeneration” (application 20140303223). Thomas Jefferson University, the author's former institute, has licensed this technology to Clementia. M.I. received a share of royalty payments from Thomas Jefferson University. NRX195183 was provided by NuRx Pharmaceuticals (Irvine, CA). 
anti-inflammatory drugs, steroids, or irradiation. ${ }^{3,15}$ These treatments are only partially effective and are accompanied by adverse effects. We have previously reported that retinoic acid receptor $\gamma(\mathrm{RAR} \gamma)$ is the dominant RAR in the regulation of chondrogenesis and that pharmacological activation of RAR $\gamma$ effectively prevents $\mathrm{HO} .{ }^{16}$ Interestingly, we have found that RAR $\gamma$ agonist not only blocks $\mathrm{HO}$ but also reduces deterioration of muscle tissue around $\mathrm{HO}$, suggesting the potential role of $\mathrm{RAR} \gamma$ in skeletal muscle damage.

Active vitamin A, retinoic acid (RA), has been known for decades to have profound effects on myogenesis. ${ }^{17,18}$ Most of its effects are mediated by nuclear RARs $(\alpha, \beta$, and $\gamma)$. On ligand binding, RAR forms a heterodimer with retinoid $\mathrm{X}$ receptors $(\alpha, \beta$, and $\gamma)$, binds to retinoic acid-responsive elements (RAREs), and regulates expression of target genes. ${ }^{19}$ In addition, RAR modulates other signaling pathways through protein-protein interactions, phosphorylation, and limiting availability of cofactors for other transcription factors. ${ }^{20}$ In the developing limb bud, RA plays an important role in recruiting muscle progenitor cells into the limb. ${ }^{21}$ Implantation of RA-soaked beads in the developing limb induces expression of muscle differentiation-related genes, such as Pax3, Myf-5, and myogenin, whereas application of citral, an inhibitor of RA signaling, downregulates early myogenic genes. ${ }^{21}$ When undifferentiated limb bud mesenchymal cells are placed in culture, RA effectively blocks chondrogenesis and stimulated myogenesis. ${ }^{22}$ On the other hand, excess RA inhibits myogenic differentiation of limb bud cells or muscle precursor cells isolated from neonatal mice. ${ }^{23,24}$ Together, these observations indicate that RA has profound effects on myogenesis, and the level of the signaling activity must be tightly controlled. However, the specific roles of individual RAR isoforms on myogenesis have not been elucidated.

Our aim was to investigate the roles of RARs in skeletal muscle injury, and then to test whether RA or its derivatives can promote muscle repair and regeneration in vivo. The results indicate the importance of endogenous RAR $\gamma$ signaling in muscle repair and a potential therapeutic value of selective $\operatorname{RAR} \gamma$ agonists for muscle injuries.

\section{Materials and Methods}

\section{Experimental Animals}

All experimental protocols involving the animals were approved by the Institutional Animal Care and Use Committee of the Jefferson Medical College and Children's Hospital of Philadelphia (Philadelphia, PA). Adult 7-weekold female CD1 mice (Charles River, Wilmington, MA) were used for all experiments unless specified. For the induction of $\mathrm{HO}$ in mice, we used the mouse model of fibrodysplasia ossificans progressiva (FOP) that carries a Cre-inducible constitutively active anaplastic lymphoma receptor tyrosine kinase (ALK) $2^{\mathrm{Q} 207 \mathrm{D}}$ mutant and enhanced green fluorescence protein. ${ }^{25}$ To induce $\mathrm{HO}$, we injected $10^{8}$ plaque-forming units $/ 10 \mu \mathrm{L}$ of Ad-Cre (Vector Biolabs, Malvern, PA) into the left tibialis anterior (TA) muscles of $\mathrm{P} 7 \mathrm{ALK} 2^{\mathrm{Q} 207 \mathrm{D}}$ mice to remove the floxed stop cassette and induce expression of mutant ALK2 gene (official name $A C V R 1)$ and then injected $10 \mu \mathrm{L}$ of $0.3 \mathrm{mg}$ cardiotoxin (C9759; Sigma-Aldrich, St. Louis, MO) to induce local inflammation in the limb. ${ }^{16}$ Retinoid signaling reporter mice [RARE-LacZ; (RARE-Hspalb/lacZ)/12Jrt/J] were obtained from the Jackson Laboratory (Bar Harbor, ME; stock number 008477).

RAR $\gamma$-null mutant mice ${ }^{26}$ were kind gifts from Dr. Pierre Chambon (INSERM, Illkirch, France). Genotyping information for the mice listed above has been previously published. ${ }^{27}$

\section{Cardiotoxin-Induced Muscle Injury Model}

The TA muscle of 7-week-old mouse was surgically exposed after anesthesia by isoflurene inhalation, and $10 \mu \mathrm{L}$ of $40 \mathrm{mmol} / \mathrm{L}$ cardiotoxin was injected into the center of the proximal $1 / 3$ level in the TA muscle. The TA muscles were collected 2 weeks after injury, fixed overnight in $4 \%$ paraformaldehyde, and subjected to histological analysis.

\section{Critical Defect Muscle Injury Model}

A round-shaped defect $(1.5-\mathrm{mm}$ diameter $\times 1.5-\mathrm{mm}$ depth) was generated with thermal cautery (model 150I; Geiger Enterprises, Council Bluffs, IA) in a TA muscle of 7-weekold female CD1 mice. Mice were then randomly divided into two groups (six mice per group) and received either vehicle or retinoids three times by oral gavage. Unless indicated, mice received retinoids on days 5, 7, and 9 after surgery, and TA muscle tissues were collected 30 days after surgery and subjected to histological analysis.

\section{Retinoid Administration}

Retinoic acid (all trans-retinoic acid, pan-RAR agonist, CAS 302-79-4) was purchased from Sigma-Aldrich (R2625). BMS270394 (RAR $\gamma$ agonist, CAS 262433-54-5) ${ }^{28}$ was obtained from Axon Medchem (Groningen, the Netherlands; Axon-1173). R667 (palovarotene, CAS410528-02-8) ${ }^{16,29}$ was synthesized at Atomax Chemicals (Shenzhen, China). NRX195183 (RAR $\alpha$ agonist) ${ }^{30}$ was a kind gift from NuRx Pharmaceuticals (Irvine, CA). CD1530 (RAR $\gamma$ agonist, CAS 107430-66-0) ${ }^{31}$ and CD437 (RAR $\gamma$ agonist, CAS 12531660-1) ${ }^{32}$ were purchased from Tocris Biosciences (Bristol, UK). The structure of NRX195183 has been shown previously ${ }^{16}$; structures of all other retinoids are shown in Supplemental Figure S1.

Stock solutions of retinoid were dissolved in dimethyl sulfoxide (D2650; Sigma-Aldrich) and stored at $-30^{\circ} \mathrm{C}$ under argon. Before administration, $30 \mu \mathrm{L}$ of stock solution was mixed with $70 \mu \mathrm{L}$ of corn oil (Sigma-Aldrich) for each dose, and delivered to mice by oral gavage. Vehicle control 
mice received $30 \mu \mathrm{L}$ dimethyl sulfoxide plus $70 \mu \mathrm{L}$ corn oil in the same manner. Control vehicle, R667, or CD1530 was administrated by oral gavage three times every other day starting at day 5 or day 8 after muscle injury surgery. This treatment regimen targets on the time point when granulation tissue actively grows and injured muscle tissues are actively remodeling. For the FOP mouse experiment, retinoid treatment was started 3 days after Ad-Cre/cardiotoxin injection. Mice received either vehicle or $4 \mathrm{mg} / \mathrm{kg}$ CD1530 every other day until the end of the experiment.

\section{Histological Analysis}

To analyze quality of muscle repair, we prepared serial transverse or longitudinal sections ( $8 \mu \mathrm{m}$ thick) of TA muscles, and stained with Masson's trichrome, according to manufacturer's protocols (Sigma-HT15; Sigma-Aldrich). Images of sections every $100 \mu \mathrm{m}$ were analyzed by ImagePro software version 7.0 (Media Cybernetics, Rockville, MD). Masson's trichrome stains muscle, adipose, and fibrous tissues in red, white, and blue, respectively. The amount of pixels of each colored area within $9 \times 9$ grids (approximately $3 \times 3 \mathrm{~mm}$ ) that includes the original injured site was quantified separately. The artificial space that was generated during tissue processing was manually subtracted from the defined $9 \times 9$ grid area to define total area. The ratio of each tissue component (pixel)/the total area (pixel) was then calculated. To optimize the image analysis procedure, we first inspected all images, identified the most severely damaged region, and preliminarily analyzed injured sites in two different ranges: three sections covering a $200-\mu \mathrm{m}$ distance [the section that has the most severely damaged region and two additional sections (100- $\mu \mathrm{m}$ apart from the first section)] and nine sections covering an $800-\mu \mathrm{m}$ distance [the section that has the most severely damaged region and eight additional sections (every 100- $\mu \mathrm{m}$ apart from the first section)]. Either analysis condition (three and nine sections) provided similar results. Therefore, we analyzed three images (the most severely damaged area in the $200-\mu \mathrm{m}$ range) per sample. To ensure objectivity of the experimental results, all image analysis was performed in a double-blinded manner (A.D.R., K.U., M.I.).

\section{Immunostaining}

After deparaffinization, slides were washed in phosphatebuffered saline and blocked in 10\% goat serum in phosphate-buffered saline. Primary antibodies [antilaminin 2, ALX-804-190 (Enzo Life Science, Farmingdale, NY), 1:250; anti-osteocalcin, M173 (Takara, Otsu, Japan), 1:500] were applied overnight at $4^{\circ} \mathrm{C}$. The secondary antibodies were applied for 1 hour and followed by counterstaining with $0.2 \mu \mathrm{g} / \mathrm{mL}$ of DAPI in phosphate-buffered saline for $10 \mathrm{mi}-$ nutes. Primary antibodies were detected with anti-rabbit Alexa Fluor 594 (Molecular Probes, Eugene, OR) and anti-rat Alexa Fluor 488-coupled secondary antibodies raised in goat
(Molecular Probes, 1:500). To detect phosphorylation of Smad1/5/8, slides were treated in citrate buffer ( $\mathrm{pH}$ 6.0) at $90^{\circ} \mathrm{C}$ for 10 minutes, blocked in $10 \%$ goat serum, and then incubated with anti-pSmad1/5/8 antibody (number 9511S; Cell Signaling, Danvars, MA; 1:200 in 1\% goat serum) for overnight at $4{ }^{\circ} \mathrm{C}$. After washing, immunoreactivity was detected by the ImmPACT Novared Kit (model SK-4805; Vector Laboratories, Burlingame, CA).

\section{Real-Time PCR}

Injured TA muscle tissues were collected and soaked in the RNAlater solution (AM7020 Ambion; Life Technologies, Grand Island, NY). The total RNA was reverse transcribed using an RT first-strand kit (330401; Qiagen, Valencia, CA). The quantification of target gene mRNA was performed by real-time PCR using SYBR Green (Life Technologies). The primer sequences are shown in Table 1.

\section{LacZ Staining}

$\beta$-Galactosidase (LacZ) activity in the TA muscles of RARE-LacZ mice was detected by Specialty Media $\beta$ Gal Staining kits (Millipore, Billerica, MA). We followed the manufacturer's protocol.

\section{Statistical Analysis}

The statistical significance of all experiments was determined by two-way factorial analysis of variance, followed by Bonferroni post hoc multiple comparison tests (Prism 5; GraphPad Software Inc., La Jolla, CA). $P<0.05$ was considered statistically significant.

\section{Results}

\section{Selective RAR $\gamma$ Agonist Inhibits Deterioration of Skeletal Muscle in FOP Model Mice}

We have recently reported that among the retinoid agonists, selective RAR $\gamma$ agonists block HO in several different HO mouse models, including the FOP model. ${ }^{16}$ In the FOP mouse model, vehicle-treated mice showed disturbance of the limb motility within a few weeks after HO induction, and X-ray analysis revealed massive HO formation (Supplemental Figure S2A). In contrast, limb movement of RAR $\gamma$ agonist (CD1530) treated mice was normal, and the X-ray image of their hind limbs had little or no evidence of HO (Supplemental Figure S2A). When we examined histology of HO in these mice, we observed that the skeletal muscle around $\mathrm{HO}$ lesion heavily deteriorated in vehicle-treated mice (Supplemental Figure S2B). In the RAR $\gamma$ agonist-treated group, disorganization of skeletal muscle tissue was much milder, although not completely normal (Supplemental Figure S2B). Parallel sections stained with anti-laminin antibody to outline the periphery of muscle fibers clearly showed differences in diameter of the 
Table 1 Primer Sequences Used in This Study

\begin{tabular}{|c|c|c|}
\hline Gene & Primer sequences & Product size (bp) \\
\hline RARA & F: 5'-GAAAAAGAAGAAAGAGGCACCCAAGC- $3^{\prime}$ & 183 \\
\hline (NM_009024) & R: 5'-AGGTCAATGTCCAGGGAGACTCGTTG-3' & \\
\hline (NM_011243) & R: 5'-GCTTGCTGGGTCGTCGTTTTCTAATG-3' & \\
\hline RARG & F: 5'-ATGGATGACACCGAGACTGGGCTACT-3' & 222 \\
\hline (NM_011244) & R: 5'-CCTTTCTGCTCCCTTAGTGCTGATGC-3' & \\
\hline$R X R \bar{B}$ & F: 5'-CGTGATAACAAAGACTGTACAGTGG-3' & 294 \\
\hline (NM_001205214) & R: 5'-GATGTTAGTCACTGGGTCATTTGG-3' & \\
\hline$R X R G$ & F: 5'-AAAGATCTCATCTACACCTGTCGG-3' & 336 \\
\hline (NM_001159731) & R: 5'-GAGGGTGAAAAGTTGCTTATCTGC-3' & \\
\hline Aldh1a1 & F: 5'-GCGTGGTAAACATTGTCCCTGGTTA-3' & 363 \\
\hline (NM_053080) & R: 5'-GCTTTGTCCAGGTTTTTGGTGAACAC-3' & \\
\hline Сур26а1 & F: 5'-GCACAAGCAGCGAAAGAAGGTGATT-3' & 278 \\
\hline (NM_007811) & R: 5'-GGAAGAGAGAAGAGATTGCGGGTCA-3' & \\
\hline Сур26b1 & F: 5'-GGCAATCTTTTTCCTCTCTCTCTTCG-3' & 385 \\
\hline (NM_001177713) & R: 5'-AACCAGTGACCAGTCTCTCCGATGAG-3' & \\
\hline Crabp1 & F: 5'-GCCAAGACGGGGATCAGTTCTACA-3' & 239 \\
\hline (NM_013496) & R: 5'-CGCCAAATGTCAGGATTAGCTCATC-3' & \\
\hline Crabp2 & F: 5'-TCTAAAGAGAAAGCCACCTTGCTGC-3' & 413 \\
\hline (NM_007759) & R: 5'-CGTCATCTGCTGTCATTGTCAGGAT-3' & \\
\hline
\end{tabular}

$F$, forward; $R$, reverse.

muscle fiber and fiber integrity between vehicle-treated and RAR $\gamma$ agonist-treated samples (Supplemental Figure S2B). There are at least two potential mechanisms by which RAR $\gamma$ agonist benefits skeletal muscle. First, RAR $\gamma$ agonist might indirectly prevent muscle degeneration by blocking $\mathrm{HO}$ because $\mathrm{HO}$ disturbs motion of the limb, leading to atrophy of skeletal muscle. Second, RAR $\gamma$ might directly affect skeletal muscle cells and/or other supporting cells in the muscle and have a beneficial action on repair of skeletal muscle damage caused by $\mathrm{HO}$ formation. We tested the latter possibility in the following experiments.

\section{RAR $\gamma$ Agonist Promotes Repair of Severely Wounded Skeletal Muscle}

To determine whether RAR $\gamma$ agonist promotes repair of injured skeletal muscle, we developed a critical muscle defect model. By using a thermal cautery, we generated a roundshaped defect (approximately 1.5-mm diameter with $1.5-\mathrm{mm}$ depth) in the TA muscle (Figure 1A). We collected injured muscle tissues at different time points after surgery and analyzed histology (Figure 1, B-M). Two days after injury, the defect was filled with exudate and blood cells (Figure 1B). The periphery of the defect was intensely stained with hematoxylin and eosin, presumably because of coagulation of proteins caused by burn injury (Figure 1F). Some muscle fibers around the defect were necrotic because they have no nuclei and typical sarcomere structure (Figure $1 \mathrm{~F}$ ). Infiltration of inflammatory cells, including leukocyte and plasma cells, was also noted (Figure 1J). Five days after surgery, the defect was filled with granulation tissue (Figure 1C). Inflammatory cell infiltration was spread between muscle fibers (Figure 1, G and K). Fourteen days after surgery, the defect was filled with a mixture of granulation and adipose tissues (Figure 1D). Immature muscle fibers appeared as characterized by presence of central nuclei (Figure 1, H and L), indicating the muscle tissue was actively remodeling and regenerating. Thirty days after surgery, the defect was mostly filled with adipose tissue (Figure 1E). Fibrous tissue was observed around the defect (Figure 1I), and immature muscle fibers were still evident (Figure 1M). At the same time point, Masson's trichrome staining of longitudinal sections showed that muscle damage was widespread and expanded along muscle fibers (Supplemental Figure S3, A-D). The defect was not restored completely for at least 2 months in three of three samples (Supplemental Figure S3E), indicating that this injury model is a critical defect model.

We used this experimental muscle injury model and examined the effects of selective RAR $\gamma$ agonists. We generated critical muscle injury in 7-week-old mice, randomly divided into three groups (six mice per group) and then treated with control vehicle, R667, or CD1530 by oral gavage at days 5,7 , and 9 after operation. This treatment regimen targets on 
A
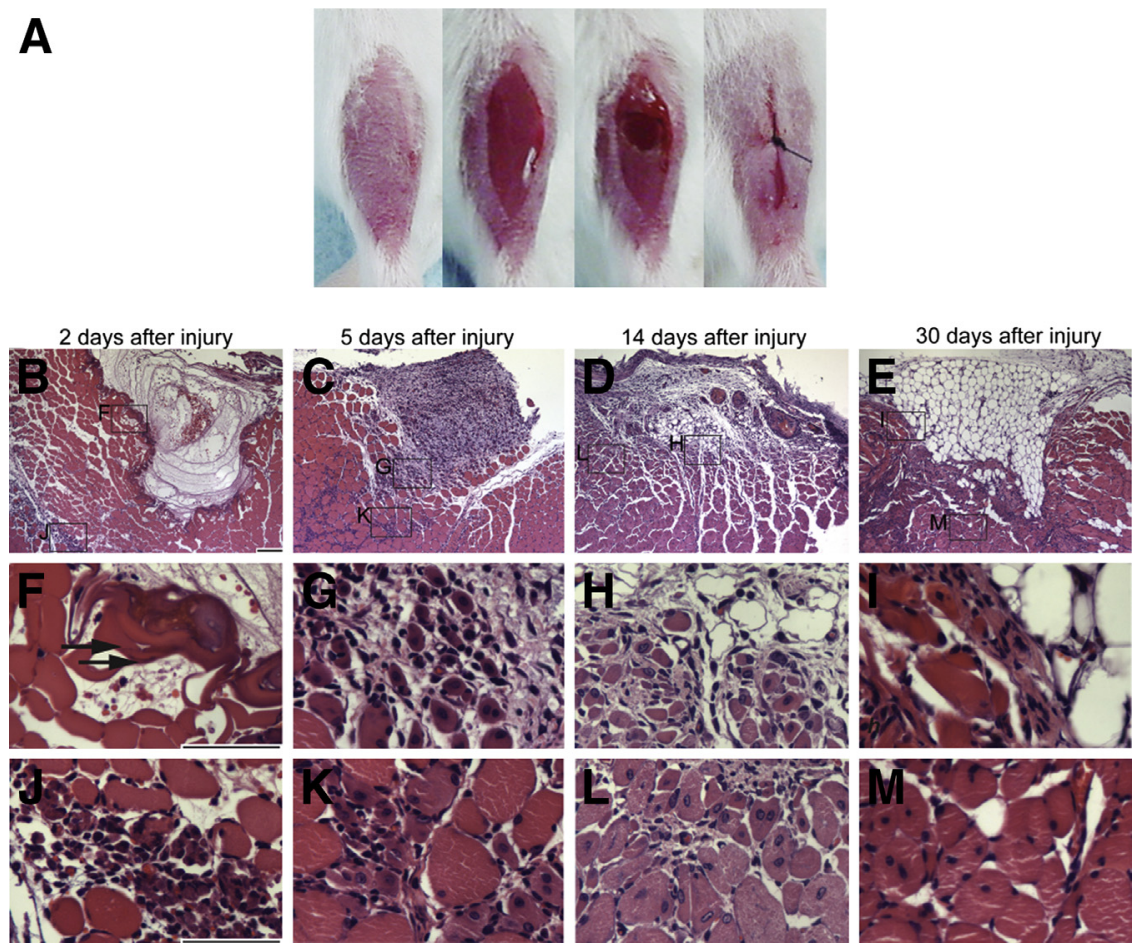

Figure 1 Critical defect muscle injury model. A: Macro images of surgical procedure for critical muscle defect injury. Tibialis anterior (TA) muscle was exposed after removal of hair and skin incision. A round defect $(1.5 \times 1.5 \mathrm{~mm})$ was then generated by a thermal cautery, and the skin was closed by suture. B-M: Histology of injured muscle after surgery. Transverse sections of injured TA muscles were prepared 2 (B, F, and $\mathbf{J}), 5$ (C, G, and $\mathbf{K}), 14(\mathbf{D}, \mathbf{H}$, and $\mathbf{L})$, and $30(\mathbf{E}, \mathbf{I}$, and $\mathbf{M})$ days after surgery and stained with hematoxylin and eosin. F-M: Magnified images of the boxed areas in $\mathbf{B}-\mathbf{E}$. Arrows in $\mathbf{F}$ indicate coagulated area. Scale bars: $100 \mu \mathrm{m}(\mathbf{B}-\mathbf{E}) ; 50 \mu \mathrm{m}(\mathbf{F}-\mathbf{M})$. the time point when granulation tissue grows and injured tissue is actively remodeling (Figure 1). Both R667 and CD1350 are RAR $\gamma$ agonists that have different back structures (Supplemental Figure S1). TA muscles were harvested 30 days after injury, fixed, and processed for histological analysis. Figure $2 \mathrm{~A}$ shows the representative transverse sections of TA muscles (three independent samples per group) that contain the center portion of the initial defects. In the control, the defect region was largely replaced by adipose and fibrous tissues. In R667- or CD1530-treated groups, the initial defect region was still identifiable, but the average size of fibrous or adipose tissue looked smaller than those in control. Inspection of serial transverse sections revealed that extension of muscle degeneration area along the longitudinal axis of the muscle and such expansion was less in RAR $\gamma$-agonist-treated groups (Figure 2B). By using the same injury model, we further examined the effects of additional selective RAR $\gamma$ agonists that have different backbone structures (Supplemental Figure S1). Operated on mice were treated with vehicle (control), R667, BMS270394, or CD437 on days 5, 7, and 9, and then analyzed 30 days after surgery (Supplemental Figure S4). The initial defect region in the control TA muscles was clearly visible as a demarcated scar, and the histology showed that the region is largely occupied by adipose tissue. In contrast, the scar tissue in the operated on region in all three RAR $\gamma$ agonist - treated groups was smaller and sometimes not visible in the macro images (Supplemental Figure S4). Because multiple RAR $\gamma$ agonists with different structures exhibit similar effects, we conclude that the promotion of muscle repair is mediated by $\operatorname{RAR} \gamma$.

To quantify the results, we performed histological image analysis. Masson's trichrome staining stained muscle, adipose, and fibrous tissues in different colors (Figure 3A). We selected each color, manually removed artificial space that was generated during the tissue processing, and measured number of pixels per defined area using ImagePro version 7.0 (Figure 3, B-D). The composition of adipose, muscle, and fibrous tissues among uninjured, vehicle-treated control, and $\operatorname{RAR} \gamma$ agonist-treated groups was compared (Figure 3E). Uninjured muscle tissue was composed of nearly $95 \%$ muscle fibers and a small percentage of adipose and fibrous tissues. The vehicle-treated group contained approximately $45 \%$ of adipose tissue and $10 \%$ of fibrous tissue. The tissue composition of the RAR $\gamma$ agonist-treated sample was not the same as that of uninjured muscle, but the ratio of adipose tissue was $15 \%$ and significantly lower in the R667 group, and 30\% in the CD1530 group (Figure 3E). The results indicate that RAR $\gamma$ agonist treatment improves the quality of muscle repair.

\section{The Effects of Other Retinoids on Muscle Repair in the} Skeletal Muscle Injury Model

To test the specificity of RAR $\gamma$ signaling to the muscle repair, we examined the effects of RA and RAR $\alpha$ agonist NRX195138 using the same muscle injury model. RA showed improvement of muscle repair in some mice (Figure 4A), whereas RAR $\alpha$ agonist did not stimulate the repair at all (Figure 4A). Histological analysis revealed that both RA and RAR $\alpha$ agonist did not significantly change the composition of muscle, adipose, and fibrous tissues in the injured site (Figure 4B).

To verify the contribution of RAR $\gamma$ signaling in skeletal muscle repair, we injured TA muscles of wild-type (WT) or RAR $\gamma$-null mice using the critical injury model or the 

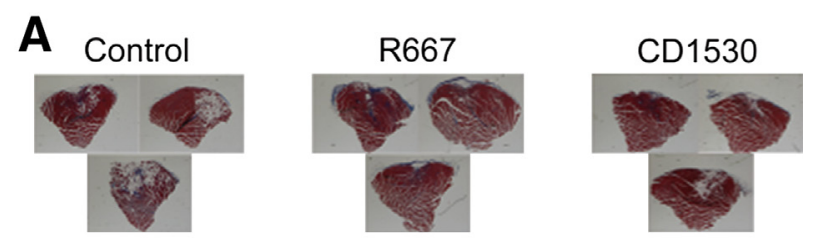

B
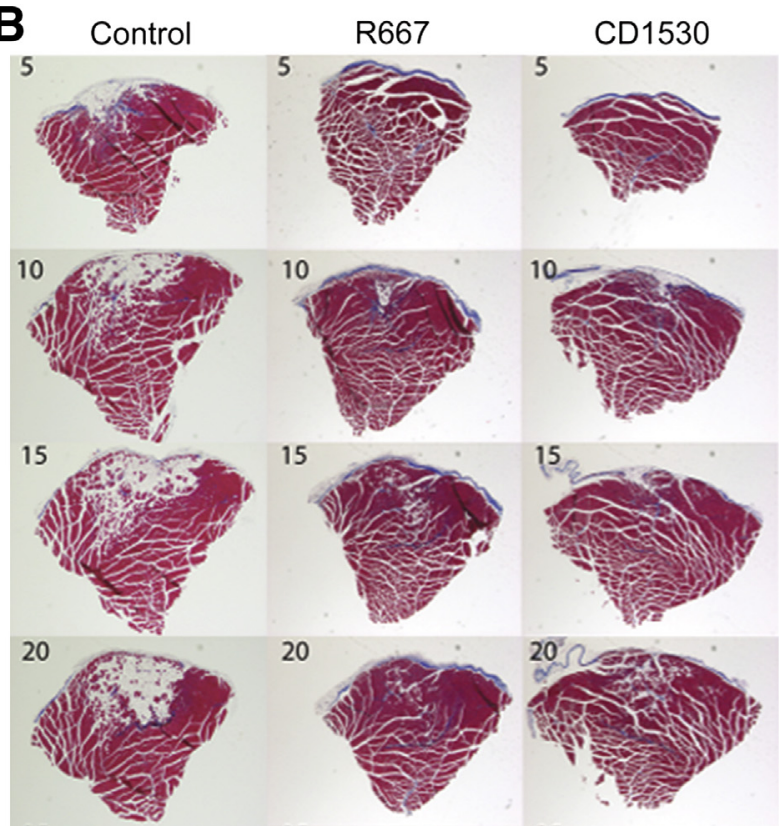

25

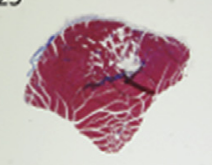

10

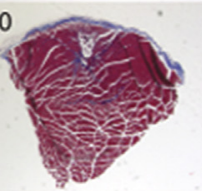

15

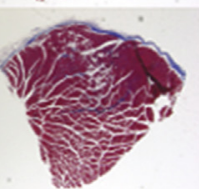

20

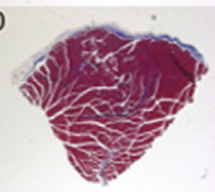

25

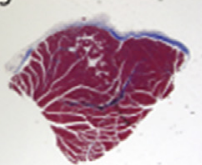

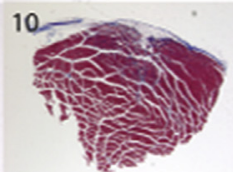
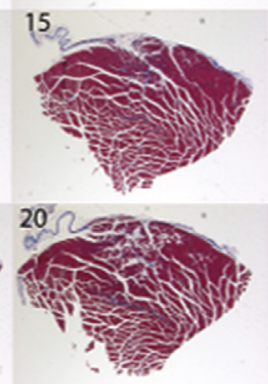

25

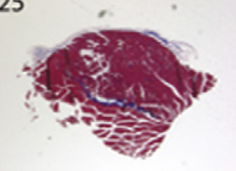

Figure 2 The effects of retinoic acid receptor $\gamma(\operatorname{RAR} \gamma)$ agonists on muscle repair in a critical defect muscle injury model. Critical defect muscle injury surgery was performed in 7-week-old female CD1 mice (six mice per group). Mice were then randomly divided and treated with vehicle, $4 \mathrm{mg} / \mathrm{kg}$ per gavage CD1530, or $4 \mathrm{mg} / \mathrm{kg}$ per gavage R667 on days 5, 7, and 9 after surgery. Muscle tissues were collected from vehicle (Control)-, R667-, and CD1530-treated mice 30 days after critical defect injury surgery. A: Representative images of transverse sections of muscles. Images that contain the largest defect were determined by visual inspection of serial sections. Three independent samples from three separated mice per group are shown. B: Representative serial transverse sections (every five slides) of muscles. The number in the upper corner indicates slide number. The interval of every five slides is approximately $1 \mathrm{~mm}$. The muscle defects were mostly replaced with the mixture of fibrous and adipose tissues. The area occupied with fibrous or adipose tissue was smaller in the RAR $\gamma$ agonisttreated sample than that of control. We repeated the experiments three times and obtained similar results. Scale bar $=1 \mathrm{~mm}(B)$.

cardiotoxin injection model. In the critical defect injury model, we observed reduction of the initial defect in the WT mice, whereas the size of the initial defect was largely unchanged in the RAR $\gamma$-null mice (Figure 4C). In the cardiotoxin injury model, the damaged area in the WT mice was fully restored by immature muscle myofibers that had central nuclei in 2 weeks (Figure 4D). In contrast, the repair process was delayed in RAR $\gamma$-null mice, as evident by the persistence of granulation tissue (Figure 4D).

Involvement of Retinoid Signaling in Muscle Repair

In the last set of experiments, we examined if retinoid signaling is active during the muscle repair process. We made an incision in the TA muscle in retinoid signaling reporter mice (RARE-LacZ mice) ${ }^{33}$ and monitored the LacZ reporter activity after muscle injury (Figure 5). In the shamoperated on mice that received skin incision but no muscle injury, we barely detected LacZ activity. In the injured muscle, LacZ activity was not detected 1 day after surgery, but was markedly up-regulated 4 and 7 days after surgery. A blue streak observed on day 4 in the sham-operated on
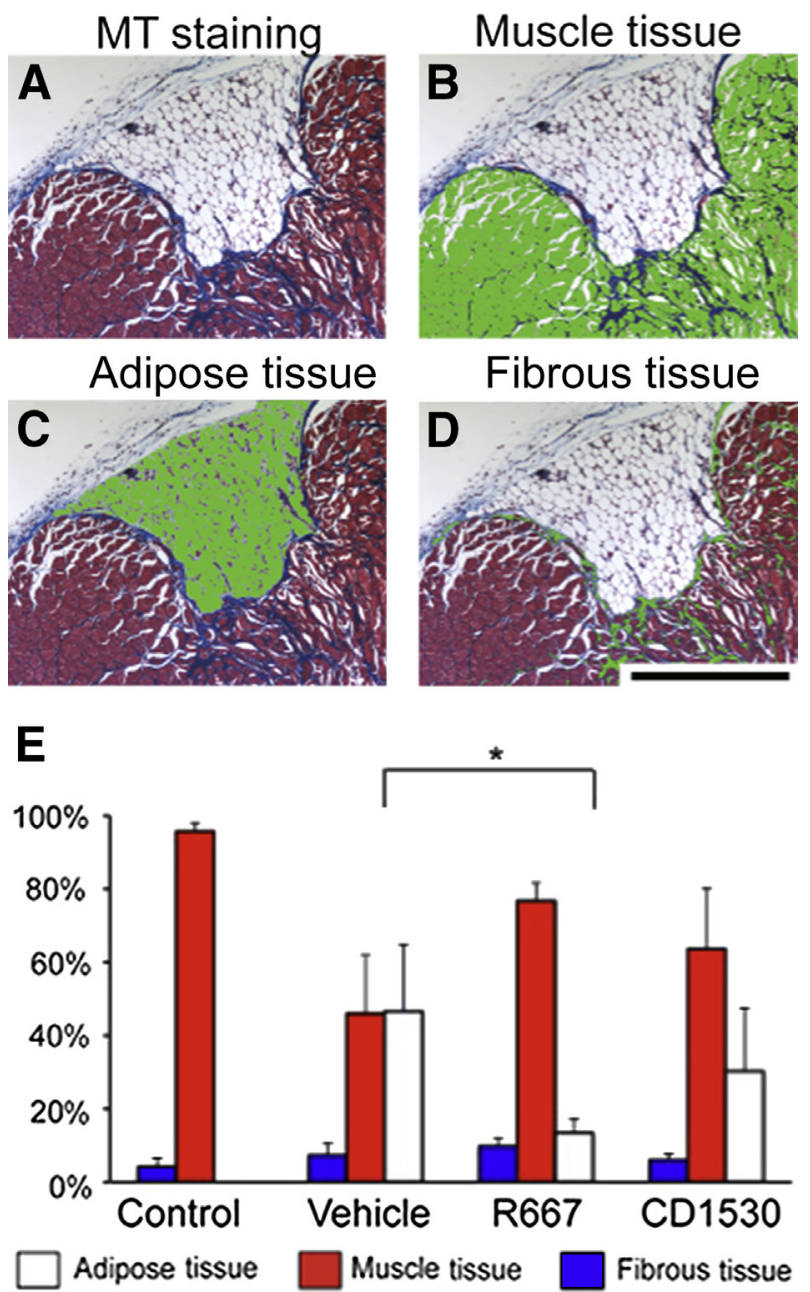

Figure 3 Histological analysis of injured muscles treated with retinoic acid receptor $\gamma$ agonists. Muscles collected from vehicle (Control)-, R667-, and CD1530-treated mice in Figure 2 were subjected to Masson's trichrome staining, followed by histological analysis. A: Image of Masson's trichrome (MT) staining. Muscle, adipose, and fibrous tissues were stained in red, white, and blue, respectively. B-D: Muscle (B), adipose (C), and fibrous (D) tissues were separately selected using Image-Pro software version 7.0. Each tissue was highlighted in green. E: Composition of adipose, muscle, and fibrous tissues in uninjured muscle (Control) or injured muscles treated with vehicle (Vehicle), CD1530, or R667. We analyzed three transverse sections covering the most severely damaged region (200- $\mu \mathrm{m}$ distance) and obtained an average value of composition for each sample. Uninjured control muscle (five mice per group) and injured muscles (six mice per group) were analyzed. Values are means \pm SD (E). ${ }^{*} P<0.05$. Scale bar $=0.5 \mathrm{~mm}(\mathbf{A}-\mathbf{D})$. 
group was probably because of the damage accidentally generated at the time of skin incision. We also collected injured and control muscle tissues after muscle injury and analyzed expressions of retinoid metabolism-related genes: Aldhla1, Aldhla2, and Aldhla3 genes encode enzymes required for biosynthesis of RA; Crabpl encodes an RA binding protein; and Cyp26al and Cyp26bl encode enzymes that metabolize RA. In the sham-operated on group,
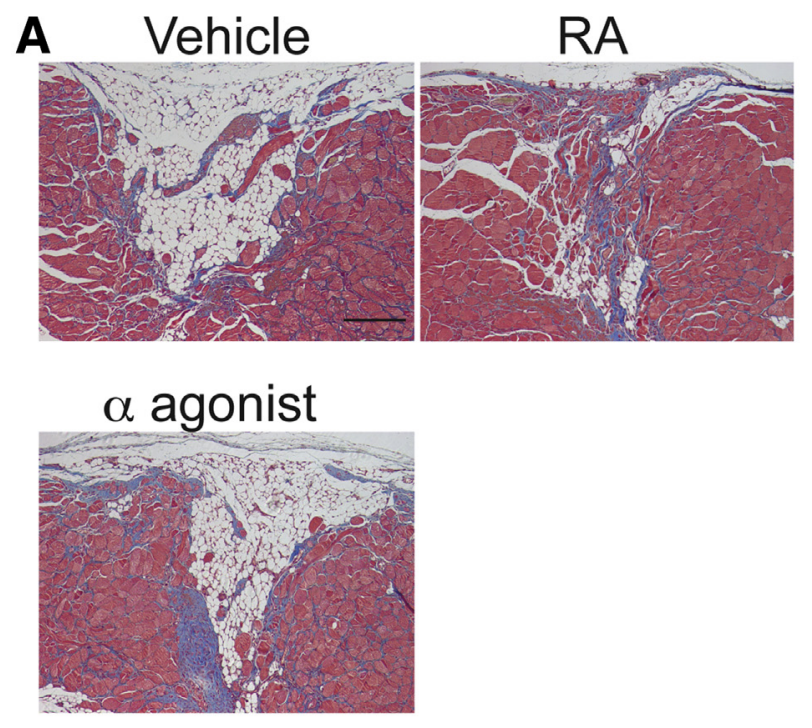

B

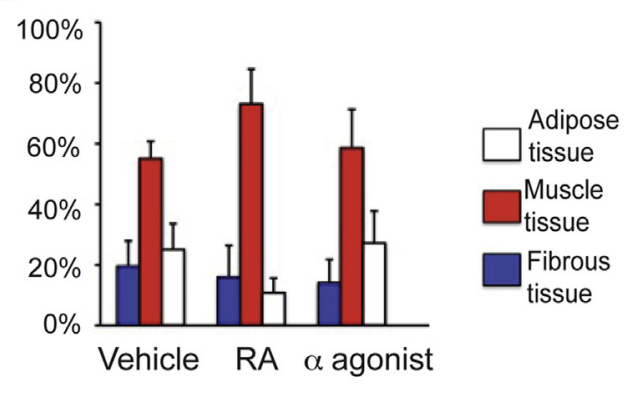

C

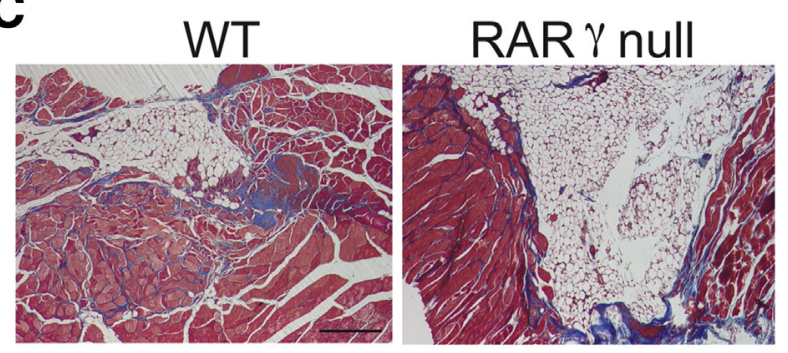

D

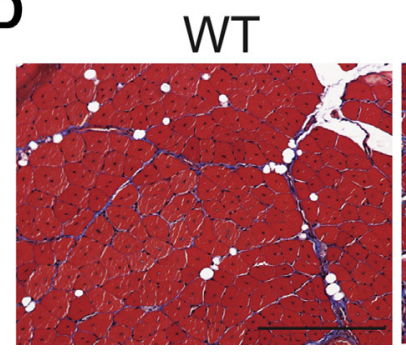

expression levels of these genes were low and did not change through the experiment. In the injured muscle, we first detected up-regulation of Crabpl, Cyp26al, and Cyp26b1, and then observed an increase in Aldhla2 gene expression. The results indicate that local RA metabolism is activated at the early stage of the muscle repair process. The specificity of primers used in real-time quantitative PCR was confirmed by regular PCR (Figure 6, A and B). We also analyzed expression of RARs and retinoid $\mathrm{X}$ receptors. The results showed that gene expression of RARs, but not retinoid $\mathrm{X}$ receptors, was up-regulated in injured muscle tissue 4 days after surgery (Figure 6C).

\section{Discussion}

In the present study, we investigated the role of retinoid signaling in the muscle repair process and demonstrated that the selective $\operatorname{RAR} \gamma$ agonist promotes muscle repair in the critical defect muscle injury model. We also observed that skeletal muscle regeneration was markedly delayed in RAR $\gamma$ null mice compared with WT mice. To our knowledge, this is the first study demonstrating that pharmacological activation of $\operatorname{RAR} \gamma$ promotes repair of injured skeletal muscle.

The results obtained from the analysis of RARE-LacZ mice revealed that local retinoid signaling is barely detectable in the intact TA muscle tissue but remarkably increased 4 days after injury (Figure 6). In parallel experiments, we examined retinoid-metabolism-related gene expression and observed up-regulation of Cyp26al and Cyp26b1 in the first 4 days after injury, followed by an increase of Aldhla 2 gene expression (Figure 6). The results indicate that local retinoid signaling is initially down-regulated after muscle injury and then up-regulated. The treatment regimen in this study targets on the time point when granulation tissue grows, injured muscle tissues are remodeling and regenerating (Figure 1), and injured muscle tissues show a high activity of retinoid signaling (Figure 5). We still do not know whether $\operatorname{RAR} \gamma$ agonists stimulate muscle repair when administrated at earlier or later stages. In the future, it is important to study the efficacy of RAR $\gamma$ agonists on muscle

\footnotetext{
Figure 4 The effects of retinoic acid (RA) and RA receptor $\alpha$ (RAR $\alpha$ ) agonist on muscle repair. $\mathbf{A}$ and $\mathbf{B}$ : Critical defect muscle injury surgery was performed in 7-week-old female CD1 mice (four mice per group). Mice were then randomly divided and treated with vehicle, $4 \mathrm{mg} / \mathrm{kg}$ per gavage RA, or $12 \mathrm{mg} / \mathrm{kg}$ per gavage RAR $\alpha$ agonist (NRX195183) on days 5, 7, and 9 after surgery. A: Injured tibialis anterior (TA) muscles of vehicle, RA, and RAR $\alpha$ agonist groups were harvested 30 days after injury and stained with Masson's trichrome. B: Compositions of adipose, muscle, and fibrous tissues were analyzed. C: Critical defect muscle injury surgery was performed in wild-type (WT) and RAR $\gamma$-null mice. Sections of injured muscles were prepared 30 days after surgery and stained with Masson's trichrome. Similar results were obtained in two independent experiments. D: WT and RAR $\gamma$-null mice (three mice per group) received cardiotoxin injection in TA muscles. Sections of TA muscles were prepared 2 weeks after injection and stained with Masson's trichrome. Muscle repair process was remarkably delayed in RAR $\gamma$-null mice. Similar results were obtained in two independent experiments. Values are means $\pm S D$ of four samples (B). $n=3$ (C). Scale bars: $0.5 \mathrm{~mm}$ (A, C, and D).
} 


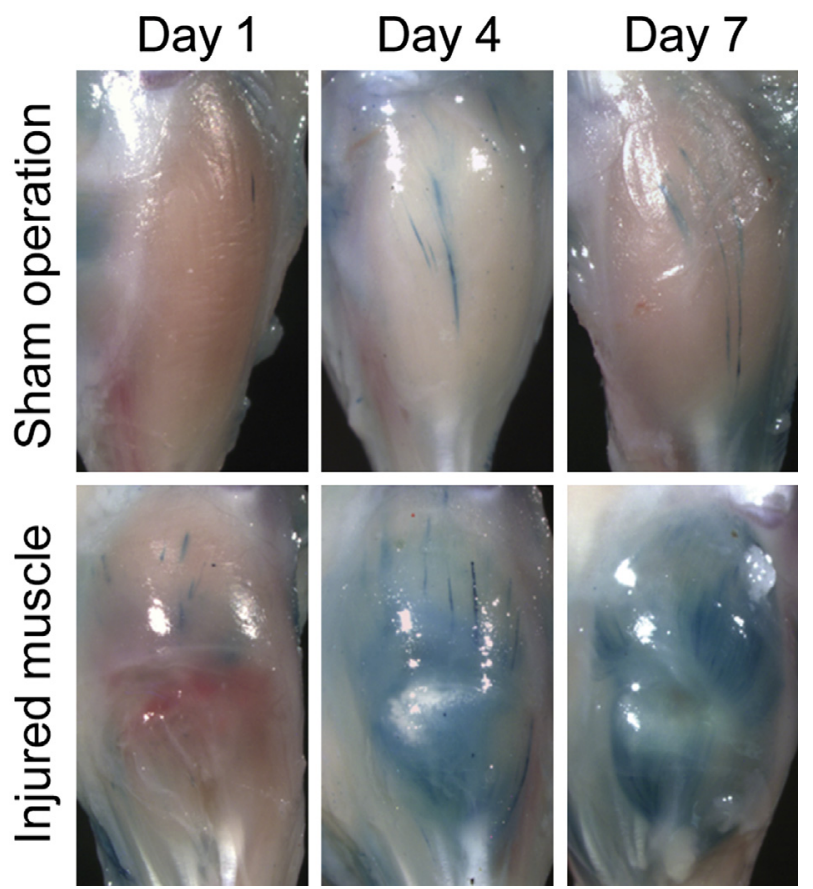

Figure 5 Up-regulation of local retinoid signaling after muscle injury. Lacerated muscle injury surgery (injured muscle) or sham surgery (only skin incision, sham operation) was performed in RARE-LacZ mice (three mice per group), as described previously. ${ }^{34}$ Muscles were collected 1, 4, and 7 days after surgery and subjected to LacZ staining. Similar results were obtained in all three mice.

repair at different time points, monitoring changes in retinoid signaling precisely.

It has been shown that bone morphogenetic protein (BMP) expression increases after muscle injury. ${ }^{35,36} \mathrm{We}$ also observed an increase in BMP signaling in injured muscle tissue, as determined by immunohistochemical staining with anti-pSmad 1,5,8 antibody (Supplemental Figure S5): a few satellite cells were pSmad1,5,8 positive in intact TA muscle, as previously reported. $^{37}$ The number of satellite cells and infiltrated inflammatory cells positive to pSmad1,5,8 remarkably increased by day 5 after surgery and gradually decreased over time; and pSmad1,5,8-positive immature muscle cells were evident 14 and 30 days after surgery. Thus, both retinoid and BMP signaling are greatly changing during muscle repair.

Several reports have shown the biological antagonism between RA and BMP, ${ }^{38-40}$ and we have reported that RAR $\gamma$, in particular, inhibits canonical BMP receptor signaling by decreasing Smad phosphorylation. ${ }^{16,41}$ From this standpoint, we think the biphasic change of local retinoid signaling may be suitable for muscle repair. The initial down-regulation of local retinoid signaling would reduce interference on BMP action by retinoid and facilitate proliferation of muscle progenitor cells. ${ }^{36}$ In addition, up-regulation of BMP signaling would facilitate recruitment of inflammatory cells that are required for tissue repair in general. ${ }^{42,43}$ On the other hand, higher RA signal is favorable for muscle cell differentiation, as shown in multiple reports. ${ }^{44-46}$ In addition, RAR $\gamma$ signaling has been reported to suppress adipogenic differentiation of
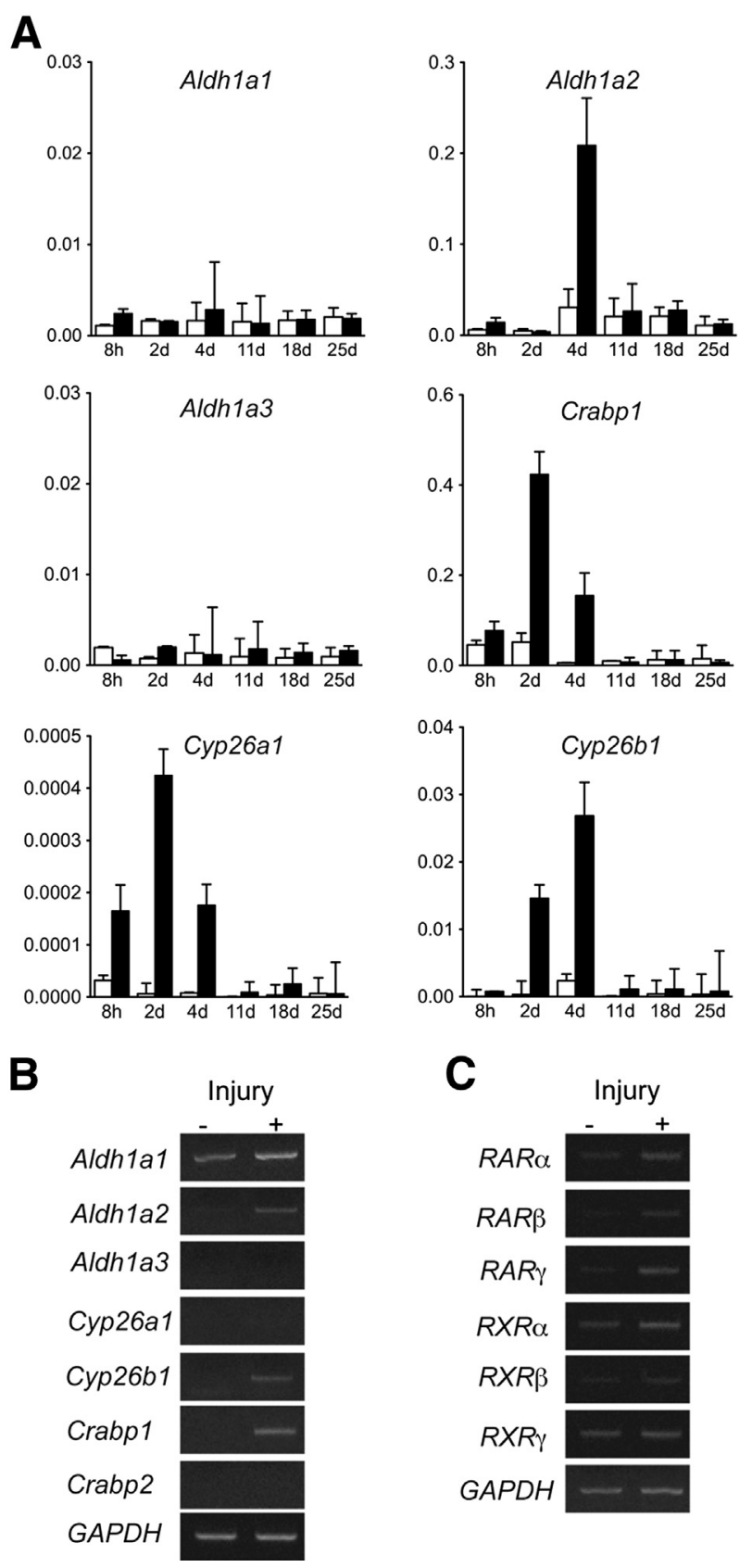

Figure 6 Gene expression of retinoic acid metabolism-related molecules and receptors in injured and intact skeletal muscle. Lacerated muscle injury surgery (injury+, black column) or sham surgery (only skin incision, injury-, white column) was performed in 7-week-old CD1 mice (four mice per group). Muscles were harvested 8 hours and 2, 4, 11, 18, or 25 days after surgery (A) or 4 days after surgery (B and $\mathbf{C})$. Total RNAs were prepared from the muscles, reverse transcribed, and subjected to real-time PCR (A) or conventional PCR (B and C). A: Changes of gene expression of retinoic acid (RA) metabolism-related enzymes after muscle injury. Graph shows relative expression levels of indicated genes to sham-operated on sample: Aldh1a1, Aldh1a2, and Aldh1a3 encode enzymes for RA biosynthesis; Crabp1 encodes an RA-binding protein; Cyp26a1 and Cyp26b1 encode enzymes that metabolize RA. B: PCR products generated with the primers that were used for real-time PCR. The results indicate specificity of these primers. C: PCR analysis of gene expression of RA receptors (RARs) in uninjured (injury - ) and injured muscle (injury + ). Values are means $\pm S D$ of four samples (A). d, days; GAPDH, glyceraldehyde-3-phosphate dehydrogenase; $h$, hours; $R X R$, retinoid $X$ receptor. 
mesenchymal stromal cells. ${ }^{47}$ Such an effect might also be a part of the mechanism for the improvement of the quality of muscle repair. Furthermore, RAR $\gamma$ signaling may be antagonizing BMP signaling in injured muscles, which would reduce the risk of $\mathrm{HO}$ in muscle.

As mentioned earlier, $\operatorname{RAR} \gamma$ regulates cell function through regulation of target genes and modulation of other signaling pathways, including BMP and Wnt signaling. ${ }^{40,41}$ Because these signaling pathways are all essential for cellular function, it is difficult to clarify the entire mechanism of RAR $\gamma$ action and to define the relative contribution of each mechanism during muscle repair. More comprehensive approaches will be required to understand RAR $\gamma$ action at the cellular level; the proteomics approach might be useful to identify RAR $\gamma$-interacting proteins, and RNA sequencing and chromatin immunoprecipitation sequencing will be required to identify $\operatorname{RAR} \gamma$ target genes.

RAR $\gamma$ agonists are small hydrophobic molecules and have several advantages over biological agents. As long as the compounds are protected from light and oxygen, RAR $\gamma$ agonists are stable, and the cost of production may be relatively low. Similar to steroids, they can be administered orally, in a tablet form, or locally, in an ointment form. One RAR $\gamma$ agonist, R667, has been tested in the phase 2 clinical trial for the treatment of the chronic lung disease, emphysema; therefore, drug safety is not an issue for this compound. Moving toward clinical trial, we have to thoroughly characterize the mechanism of action of RAR $\gamma$ in skeletal muscle and also optimize the treatment regimen. The efficacy of the drug should also be confirmed in large animals. Nevertheless, we believe that selective RAR $\gamma$ agonists will be effective drugs for the treatment of severe muscle injury and degeneration.

\section{Acknowledgments}

We thank Dr. Kengo Shimono (Okayama University Graduate School of Medicine, Dentistry and Pharmaceutical Sciences, Okayama, Japan) for providing protocols and technical advice for experimental muscle injury models; and Drs. Pierre Chambon and Norbert Ghyselinck (INSERM, Illkirch Cedex, France) for providing retinoic acid receptor $\gamma$-null mice.

M.I. directed the project. A.D.R. and M.I. wrote the manuscript. A.D.R. and K.U. performed mouse operation and treatments and gene expression analysis. C.L. and R.B. analyzed histology. E.R.B. provided expertise on muscle biology. All authors analyzed data.

\section{Supplemental Data}

Supplemental material for this article can be found at http://dx.doi.org/10.1016/j.ajpath.2015.05.007.

\section{References}

1. Close GL, Kayani A, Vasilaki A, McArdle A: Skeletal muscle damage with exercise and aging. Sports Med 2005, 35:413-427
2. Aarimaa V, Rantanen J, Heikkila J, Helttula I, Orava S: Rupture of the pectoralis major muscle. Am J Sports Med 2004, 32:1256-1262

3. McCarthy EF, Sundaram M: Heterotopic ossification: a review. Skeletal Radiol 2005, 34:609-619

4. Rantanen J, Thorsson O, Wollmer P, Hurme T, Kalimo H: Effects of therapeutic ultrasound on the regeneration of skeletal myofibers after experimental muscle injury. Am J Sports Med 1999, 27:54-59

5. Almekinders LC: Anti-inflammatory treatment of muscular injuries in sport: an update of recent studies. Sports Med 1999, 28:383-388

6. Song YH, Song JL, Delafontaine P, Godard MP: The therapeutic potential of IGF-I in skeletal muscle repair. Trends Endocrinol Metab 2013, 24:310-319

7. Musaro A, Giacinti C, Borsellino G, Dobrowolny G, Pelosi L, Cairns L, Ottolenghi S, Cossu G, Bernardi G, Battistini L, Molinaro M, Rosenthal N: Stem cell-mediated muscle regeneration is enhanced by local isoform of insulin-like growth factor 1. Proc Natl Acad Sci U S A 2004, 101:1206-1210

8. Musaro A, McCullagh K, Paul A, Houghton L, Dobrowolny G, Molinaro M, Barton ER, Sweeney HL, Rosenthal N: Localized Igf-1 transgene expression sustains hypertrophy and regeneration in senescent skeletal muscle. Nat Genet 2001, 27:195-200

9. Pelosi L, Giacinti C, Nardis C, Borsellino G, Rizzuto E, Nicoletti C, Wannenes F, Battistini L, Rosenthal N, Molinaro M, Musaro A: Local expression of IGF-1 accelerates muscle regeneration by rapidly modulating inflammatory cytokines and chemokines. FASEB J 2007, 21:1393-1402

10. Krivickas LS, Walsh R, Amato AA: Single muscle fiber contractile properties in adults with muscular dystrophy treated with MYO-029. Muscle Nerve 2009, 39:3-9

11. Chalmers J, Gray DH, Rush J: Observations on the induction of bone in soft tissues. J Bone Joint Surg Br 1975, 57:36-45

12. Garland DE: A clinical perspective on common forms of acquired heterotopic ossification. Clin Orthop Relat Res 1991, (263):13-29

13. Nilsson OS, Persson PE: Heterotopic bone formation after joint replacement. Curr Opin Rheumatol 1999, 11:127-131

14. Forsberg JA, Pepek JM, Wagner S, Wilson K, Flint J, Andersen RC, Tadaki D, Gage FA, Stojadinovic A, Elster EA: Heterotopic ossification in high-energy wartime extremity injuries: prevalence and risk factors. J Bone Joint Surg Am 2009, 91:1084-1091

15. Nauth A, Giles E, Potter BK, Nesti LJ, O'Brien FP, Bosse MJ, Anglen JO, Mehta S, Ahn J, Miclau T, Schemitsch EH: Heterotopic ossification in orthopaedic trauma. J Orthop Trauma 2012, 26: 684-688

16. Shimono K, Tung WE, Macolino C, Chi AH, Didizian JH, Mundy C, Chandraratna RA, Mishina Y, Enomoto-Iwamoto M, Pacifici M, Iwamoto M: Potent inhibition of heterotopic ossification by nuclear retinoic acid receptor-gamma agonists. Nat Med 2011, 17:454-460

17. Ryan T, Liu J, Chu A, Wang L, Blais A, Skerjanc IS: Retinoic acid enhances skeletal myogenesis in human embryonic stem cells by expanding the premyogenic progenitor population. Stem Cell Rev 2012, 8:482-493

18. Hamade A, Deries M, Begemann G, Bally-Cuif L, Genet C, Sabatier F, Bonnieu A, Cousin X: Retinoic acid activates myogenesis in vivo through Fgf8 signalling. Dev Biol 2006, 289:127-140

19. Duester G: Retinoic acid synthesis and signaling during early organogenesis. Cell 2008, 134:921-931

20. Rochette-Egly C, Germain P: Dynamic and combinatorial control of gene expression by nuclear retinoic acid receptors (RARs). Nucl Recept Signal 2009, 7:e005

21. Reijntjes S, Francis-West P, Mankoo BS: Retinoic acid is both necessary for and inhibits myogenic commitment and differentiation in the chick limb. Int J Dev Biol 2010, 54:125-134

22. Pacifici M, Cossu G, Molinaro M, Tato F: Vitamin A inhibits chondrogenesis but not myogenesis. Exp Cell Res 1980, 129:469-474

23. Robson LG, Kara T, Crawley A, Tickle C: Tissue and cellular patterning of the musculature in chick wings. Development 1994, 120 : $1265-1276$ 
24. Xiao Y, Grieshammer U, Rosenthal N: Regulation of a muscle-specific transgene by retinoic acid. J Cell Biol 1995, 129:1345-1354

25. Fukuda T, Scott G, Komatsu Y, Araya R, Kawano M, Ray MK, Yamada M, Mishina Y: Generation of a mouse with conditionally activated signaling through the BMP receptor, ALK2. Genesis 2006, 44:159-167

26. Chapellier B, Mark M, Garnier JM, Dierich A, Chambon P, Ghyselinck NB: A conditional floxed (loxP-flanked) allele for the retinoic acid receptor gamma (RARgamma) gene. Genesis 2002, 32:95-98

27. Williams JA, Kondo N, Okabe T, Takeshita N, Pilchak DM, Koyama E, Ochiai T, Jensen D, Chu ML, Kane MA, Napoli JL, Enomoto-Iwamoto M, Ghyselinck N, Chambon P, Pacifici M, Iwamoto M: Retinoic acid receptors are required for skeletal growth, matrix homeostasis and growth plate function in postnatal mouse. Dev Biol 2009, 328:315-327

28. Klaholz BP, Mitschler A, Belema M, Zusi C, Moras D: Enantiomer discrimination illustrated by high-resolution crystal structures of the human nuclear receptor hRARgamma. Proc Natl Acad Sci U S A 2000, 97:6322-6327

29. Brennan BJ, Brown AB, Kolis SJ, Rutman O, Gooden C, Davies BE: Effect of R667, a novel emphysema agent, on the pharmacokinetics of midazolam in healthy men. J Clin Pharmacol 2006, 46:222-228

30. Shimono K, Morrison TN, Tung WE, Chandraratna RA, Williams JA, Iwamoto M, Pacifici M: Inhibition of ectopic bone formation by a selective retinoic acid receptor alpha-agonist: a new therapy for heterotopic ossification? J Orthop Res 2010, 28:271-277

31. Thacher SM, Vasudevan J, Chandraratna RA: Therapeutic applications for ligands of retinoid receptors. Curr Pharm Des 2000, 6:25-58

32. Zhao X, Spanjaard RA: The apoptotic action of the retinoid CD437/AHPN: diverse effects, common basis. J Biomed Sci 2003, 10: 44-49

33. Rossant J, Zirngibl R, Cado D, Shago M, Giguere V: Expression of a retinoic acid response element-hsplacZ transgene defines specific domains of transcriptional activity during mouse embryogenesis. Genes Dev 1991, 5:1333-1344

34. Menetrey J, Kasemkijwattana C, Fu FH, Moreland MS, Huard J: Suturing versus immobilization of a muscle laceration: a morphological and functional study in a mouse model. Am J Sports Med 1999, 27: 222-229

35. Clever JL, Sakai Y, Wang RA, Schneider DB: Inefficient skeletal muscle repair in inhibitor of differentiation knockout mice suggests a crucial role for BMP signaling during adult muscle regeneration. Am J Physiol Cell Physiol 2010, 298:C1087-C1099
36. Friedrichs M, Wirsdoerfer F, Flohe SB, Schneider S, Wuelling M, Vortkamp A: BMP signaling balances proliferation and differentiation of muscle satellite cell descendants. BMC Cell Biol 2011, 12:26

37. Sartori R, Schirwis E, Blaauw B, Bortolanza S, Zhao J, Enzo E, Stantzou A, Mouisel E, Toniolo L, Ferry A, Stricker S, Goldberg AL, Dupont S, Piccolo S, Amthor H, Sandri M: BMP signaling controls muscle mass. Nat Genet 2013, 45:1309-1318

38. Weston AD, Rosen V, Chandraratna RA, Underhill TM: Regulation of skeletal progenitor differentiation by the BMP and retinoid signaling pathways. J Cell Biol 2000, 148:679-690

39. Garnaas MK, Cutting CC, Meyers A, Kelsey PB Jr, Harris JM, North TE, Goessling W: Rargb regulates organ laterality in a zebrafish model of right atrial isomerism. Dev Biol 2012, 372:178-189

40. Hoffman LM, Garcha K, Karamboulas K, Cowan MF, Drysdale LM, Horton WA, Underhill TM: BMP action in skeletogenesis involves attenuation of retinoid signaling. J Cell Biol 2006, 174:101-113

41. Sheng N, Xie Z, Wang C, Bai G, Zhang K, Zhu Q, Song J, Guillemot F, Chen YG, Lin A, Jing N: Retinoic acid regulates bone morphogenic protein signal duration by promoting the degradation of phosphorylated Smad1. Proc Natl Acad Sci U S A 2010, 107: 18886-18891

42. Raida M, Heymann AC, Gunther C, Niederwieser D: Role of bone morphogenetic protein 2 in the crosstalk between endothelial progenitor cells and mesenchymal stem cells. Int J Mol Med 2006, 18: $735-739$

43. Fiedler J, Roderer G, Gunther KP, Brenner RE: BMP-2, BMP-4, and PDGF-bb stimulate chemotactic migration of primary human mesenchymal progenitor cells. J Cell Biochem 2002, 87:305-312

44. Mok GF, Cardenas R, Anderton H, Campbell KH, Sweetman D: Interactions between FGF18 and retinoic acid regulate differentiation of chick embryo limb myoblasts. Dev Biol 2014, 396:214-223

45. Yuan J, Tang Z, Yang S, Li K: CRABP2 promotes myoblast differentiation and is modulated by the transcription factors MyoD and $\mathrm{Sp} 1$ in C2C12 cells. PLoS One 2013, 8:e55479

46. Zhu GH, Huang J, Bi Y, Su Y, Tang Y, He BC, He Y, Luo J, Wang Y, Chen L, Zuo GW, Jiang W, Luo Q, Shen J, Liu B, Zhang WL, Shi Q, Zhang BQ, Kang Q, Zhu J, Tian J, Luu HH, Haydon RC, Chen Y, He TC: Activation of RXR and RAR signaling promotes myogenic differentiation of myoblastic C2C12 cells. Differentiation 2009, 78: 195-204

47. Berry DC, DeSantis D, Soltanian H, Croniger CM, Noy N: Retinoic acid upregulates preadipocyte genes to block adipogenesis and suppress diet-induced obesity. Diabetes 2012, 61:1112-1121 